\title{
Ueber einige Doppelsalze des salpetersauren Silbers mit Jod-, Brom- und Chlorsilber;
}

\section{von Hugo Risse,}

Assistent am chemischen Laboratorium der polytechnischen Schule zu Carlsruhe.

Professor Weltzien theilte in diesen Annalen XCV, 227 die von den Herren Tobler und Stamm ausgefürten Analysen eines Doppelsalzes von Jodsilber mit salpetersaurem Silber mit, welches aus einer mit Jodsilber heifs gesättigten Lösung von salpetersaurem Silber herauskrystallisirt war. Nach diesen konnte für die Verbindung nur die Formel AgJ +2 AgNO $^{6}$ entwickelt werden. Gleichzeitig beobachtete Schnaufs*) die Bildung einer krystallisirbaren Verbindung dieser zwei Körper, für welche er die Formel AgJ + AgNO $^{6}$ aufstellte. Endlich giebt Kremer**) an, dafs ihm die Darstellung der Weltzien'schen Verbindung nicht gelungen sei, während er ein krystallinisches, der Schnaufs'schen Verbindung analog zusammengeseiztes Salz darstellte ${ }^{* * *}$ ).

*) Archir d. Pharm. LXXXIr, 260.

**) Journ. für pract. Chemie LXXI, 54.

***) Unbekannt mit diesen Untersuchungen hat A. Riche im verflossenen Jahre (J. pharm. [3] XXXIII, 343) die Einwirkung des salpetersauren Silbers auf Jod-, Brom- und Chlorsilber untersucht. Durch Kochen einer mälsig concentrirten Lösung von salpetersaurem Silber mit Jodsilber und Erkaltenlassen der heifs filtrirten Flüssigkeit erhielt er eine Verbindung, bei langsamerem Krystallisiren aus verdünnterer Flüssigkeit in Form langer farbloser Nadeln, aus concentrirter Flüssigkeit hingegen in Form kleiner farbloser Schuppen, mit der von Weltzien angegebenen Zusammensetzung AgJ + $2 \mathrm{AgNO}^{6}$. Die Verbindung gab bei der Zersetzung mit Wasser 40,06 u. 40,19 pC. Jodsilber, 
Professor Weltzien veranlafste mich, die Untersuchung wieder aufzunehmen, deren Ergebnisse ich hier kurz mittheile.

Salpetersaures Silber-Jodsilber. - Wird eine heifse concentrirte Lösung von salpetersaurem Silber mit überschüssigem Jodsilber gekocht, so löst sich dieses in reichlicher Menge auf, und es scheidet sich, je nach der Menge des Jodsilbers, eine hell- bis braungelbe ölartige Flüssigkeit am Boden ab. Aus der obenstehenden klaren Flüssigkeit krystallisirt beim Erkalten eine Verbindung von Jodsilber mit salpetersaurem Silber in wohl ausgebildeten Prismen, oder in concentrisch gruppirten flachen Aggregationen aus. Noch leichter und in gröfserer Menge erhält man diese Verbindung durch Zusammenschmelzen von salpetersaurem Silber mit $1 / 2$ Aeq. Jodsilber. Die Masse schmilzt sehr leicht $\mathrm{zu}$ einer klaren, hellgelben, durchsichtigen Flüssigkeit, welche beim Erkalten krystallinisch erstarrt. Behandelt man diese mit wenig siedendem Wasser, so löst sich ein Theil auf,

und in Lösung blieb dabei so viel Silber als 58,85 n. 59,74 pC. salpetersaurem Silber entspricht. Nach Riche bildet sich bei längerem Kochen einer angemessen verdünnten Lösung von salpetersaurem Silber mit Bromsilber eine Verbindung beider Salze, die in seideartigen Nadeln krystallisire, aber äufserst leicht zersetzbar und nicht wohl rein zu erhalten sei (ein Präparat ergab 50,06 , ein anderes 47,76 pC. Bromsilber; die Verbindung sei so wenig beständig, dals gleich nach ihrer Bildung ein Theil des Bromsilbers sich ausscheide und der noch unzersetzt auskrystallisirenden Verbindung beimenge); Schna Is und Kremer hatten eine solche Verbindung auf nassem Wege nicht erhalten. Chlorsilber verbindet sich auch nach $R$ iche's Versuchen, wie nach denen der genannten Chemiker, mit salpetersaurem Silber nicht, sondern aus der durch Kochen des ersteren mit einer Lösung des letzteren erhaltenen Flüssigkeit krystallisirt bei dem Erkalten fast reines Chlorsilber.

D. $\boldsymbol{R}$. 
während ein anderer Theil als öliger Körper am Boden zurückbleibt. Aus dieser Auflösung erhält man bei langsamem Erkalten prachtvolle Krystallgruppen des Doppelsalzes.

Die auf die eine oder die andere Art erhaltene Verbindung bildet schöne seideglänzende farblose Krystalle, die sich am Licht ziemlich rasch schwärzen. Durch viel Wasser und Alkohol werden sie zersetzt, jedoch nur schwierig vollständig, indem das sich ausscheidende Jodsilber die Krystalle umhüllt und so die Einwirkung des Wassers erschwert. In wenig siedendem Wasser sind sie auflöslich. Sie schmelzen bei $105^{\circ}$, erstarren aber erst bei $98^{\circ}$ und unter Umständen noch später, wobei das Thermometer wieder auf $105^{\circ}$ steigt.

I. 0,9172 Grm. gaben 0,3769 Grm. AgJ und 0,4609 Grm. $\mathrm{AgCl}$, entsprechend $0,5460 \mathrm{Grm}$. $\mathrm{AgNO}^{6}$.

II. 0,7973 Grm. gaben 0,3208 Grm. AgJ und 0,3976 Grm. $\mathrm{AgCl}$, entsprechend $0,4710 \mathrm{Grm} . \mathrm{AgNO}^{6}$.

\begin{tabular}{cccccc} 
& \multicolumn{2}{c}{} & \multicolumn{2}{c}{ Gefunden } \\
& \multicolumn{2}{c}{ Berechnet } & & I. & II. \\
2 AgNO $^{6}$ & 340 & 59,13 & & 59,53 & 59,07 \\
AgJ & $\mathbf{2 3 5}$ & 40,87 & & 41,09 & 40,24 \\
\cline { 2 - 3 } \cline { 5 - 6 } & 575 & 100,00 & & 100,62 & $99,31$.
\end{tabular}

Was die ölartige Flüssigkeit, welche sich bei der Darstellung dieses Salzes am Boden abschied, betrifft, so ist dieselbe verschieden zusammengesetzt, je nach der gröfseren oder geringeren Menge des angewandten Jodsilbers. Durch fortgesetztes Kochen mit einer Lösung von salpetersaurem Silber wird so lange noch Jodsilber ausgezogen, bis die Masse nur noch schwach gelblich gefärbt ist. Alsdann hat sich eine constante Verbindung gebildet, welche vollständig in wenig siedendem Wasser aufgelöst werden kann. Giefst man nach dem Erstarren dieser geschmolzenen Masse die 
überstehende Flüssigkeit ab und trocknet zwischen Fliefspapier, so erhält man ein Doppelsalz, welches die Zusammensetzung und die Eigenschaften der vorhin beschriebenen Verbindung zeigt.

I. 0,1394 Grm. gaben 0,05603 Grm. AgJ.

Il. $0,9011 \mathrm{Grm}$. gaben $0,3605 \mathrm{Grm}$. $\mathrm{AgJ}$ und $0,4523 \mathrm{Grm}$. $\mathrm{AgCl}$, entsprechend $0,5358 \mathrm{Grm}$. $\mathrm{AgNO}^{6}$.

\begin{tabular}{ccccc} 
& \multicolumn{2}{c}{ Berechnet } & \multicolumn{2}{c}{ Gefunden } \\
\cline { 3 - 5 } $2 \mathrm{AgNO}^{6}$ & 340 & 59,13 & I. & II. \\
$\mathrm{AgJ}$ & 235 & 40,87 & 40,19 & 40,46 \\
\end{tabular}

Die von Kremer beschriebene Verbindung habe ich durch Auflösung von Jodsilber in salpetersaurem Silber als constante Verbindung nicht erhalten können; es scheint dieselbe eine Auflösung von Jodsilber in dem geschmolzenen Doppelsalze $2 \mathrm{AgNO}^{6}+\mathrm{AgJ}$ zu sein, wie dieses $\mathrm{Salz}$ anch noch grölsere Mengen Jodsilber als 1 Aeq. aufzulösen im Stande ist.

Salpetersaures Silber-Bromsilber. - Frisch gefälltes Bromsilber löst sich, wenn auch nur schwierig, in heifser, sehr concentrirter Lösung von salpetersaurem Silber auf, und es scheiden sich beim Erkalten feine Krystalle einer Verbindung dieser Körper aus. Es ist nöthig, die Krystalle vor dem vollständigen Erkalten der Lösung von der Mutterlauge zu trennen, da sie sonst mit Krystallen von salpetersaurem Silber vermengt werden. Die Multerlauge vermag neue Mengen von Bromsilber aufzulösen.

0,6531 Grm. gaben 0,3405 Grm. $\mathrm{AgBr}$ und 0,2635 Grm. $\mathrm{AgCl}$, entsprechend $0,3150 \mathrm{Grm}$. AgNO ${ }^{6}$.

\begin{tabular}{lccc} 
& \multicolumn{2}{c}{ Berechnet } & Gefunden \\
$\mathrm{AgN0}^{6}$ & 170 & $\mathbf{4 7 , 4 8}$ & $\mathbf{4 7 , 8 0}$ \\
$\mathrm{AgBr}$ & $\mathbf{1 8 8}$ & $\mathbf{5 2 , 5 2}$ & $\mathbf{5 1 , 9 8}$ \\
\hline & $\mathbf{3 5 8}$ & $\mathbf{1 0 0 , 0 0}$ & $\mathbf{9 9 , 7 8}$.
\end{tabular}


Das salpetersaure Silber-Bromsilber besitzt also die Formel $\mathrm{AgNO}^{6}+\mathrm{AgBr}$. Es bildet harte, farblose, seideglänzende prismatische Säulen, welche sich am Licht ziemlich rasch schwärzen und von Wasser und Alkohol sogleich unter Ausscheidung von Bromsilber zersetzt werden, wobei man schöne Pseudomorphosen von Bromsilber nach dieser Verbindung erhält. Die Krystalle schmelzen bei $175^{\circ}$ zu einer beim Erkalten krystallinisch erstarrenden gelblichen Masse.

Salpetersaures Silber-Chlorsilber. - Noch schwieriger als Bromsilber löst sich Chlorsilber in einer siedenden gesättigten Lösung von salpetersaurem Silber. Giefst man nach längerem Kochen die Flüssigkeit vom ungelösten Chlorsilber ab, so scheiden sich zuweilen in der noch heifsen Lösung feine prismatische Krystalle aus, welche wahrscheinlich eine an Chlorsilber reichere Verbindung der beiden Körper ist. Es gelang mir nicht, dieselben von der überstehenden Flüssigkeit zu trennen, indem der geringste Stols schon ein Erstarren der ganzen Flüssigkeit zu einem feinen Krystallbrei verursachte. Prefst man diesen Krystallbrei zwischen Fliefspapier ab, so erhält man farblose, unter dem Mikroscop vollständig homogen erscheinende Krystalle, welche bei $186^{\circ}$ schmelzen. Sie sind gegen das Licht ziemlich indifferent und werden von viel Wasser zerselzt, während sie sich in wenig kochendem Wasser lösen. Bei der Analyse der Verbindung von verschiedenen Darstellungen gaben :

I. 1,4832 Grm. 0,0669 Grm. AgCl, entsprechend 4,51 pC., und II. $1,2496,0,0603, \quad, \quad, \quad 4,83$ pC., welche Zusammensetzung am nächsten der Formel $18 \mathrm{AgNO}^{6}$ + $\mathrm{AgCl}$ entspricht. Doch ist es wohl fraglich, ob überhaupt hier ein stöchiometrisches Verhältnifs staltfindet.

Carlsruhe im April 1859. 